\title{
Metamodeling methods that incorporate qualitative variables for improved design of vegetative filter strips.
}

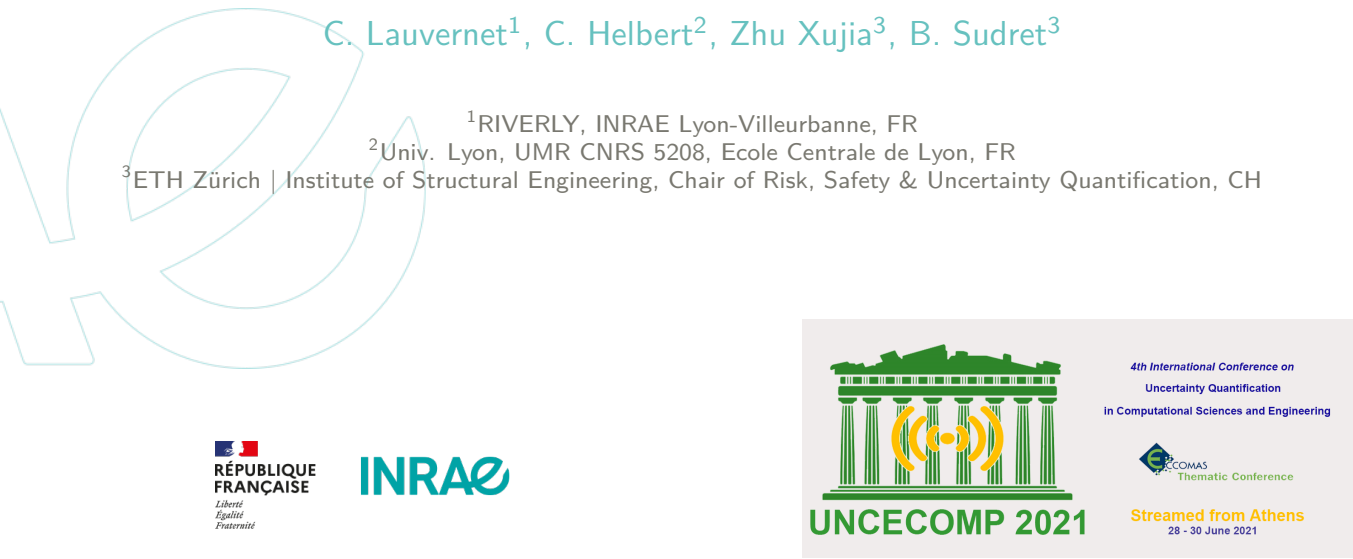




\section{Mitigation of non-point source inputs in France and in EU}

- Significant amounts of pollutants are measured in surface water

- Vegetative filter strips (VFS) are identified as the BMP of Choice for Runoff mitigation

- VFSs are mandatory or advised depending on the country and conditions

- They need to be properly designed, considering the specific context

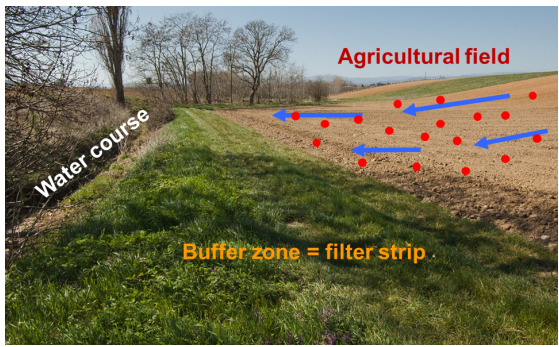




\section{Mitigation of non-point source inputs in France and in EU}

- Significant amounts of pollutants are measured in surface water

- Vegetative filter strips (VFS) are identified as the BMP of Choice for Runoff mitigation

- VFSs are mandatory or advised depending on the country and conditions

- They need to be properly designed, considering the specific context

Development of a specific tool to design VFS, once a local diagnosis has been realized:

BUffer strip for runoff Attenuation and pesticides Retention Design tool

${ }^{a}$ Carluer, N., Lauvernet, C., Noll, D, Muñoz-Carpena, R. Defining context-specific scenarios to design vegetated buffer zones that limit pesticide transfer via surface runoff Sc. of The Total Env., 2017, 575, 701-712 


\section{BUVARD issues for operational purposes}

- Processes that drive the pesticide fate at the catchment scale are complex and interact : infiltration, surface runoff, sediment trapping, pesticide transfer, etc.

- BUVARD is in fact a chain of several models

- their description is based on non linear equations and/or conceptual and/or stochastic

- a large set of parameters that are difficult to measure/estimate

- inputs and outputs are dynamic (ex : rainfall)

$\Rightarrow$ a high uncertainty in an operationnal context

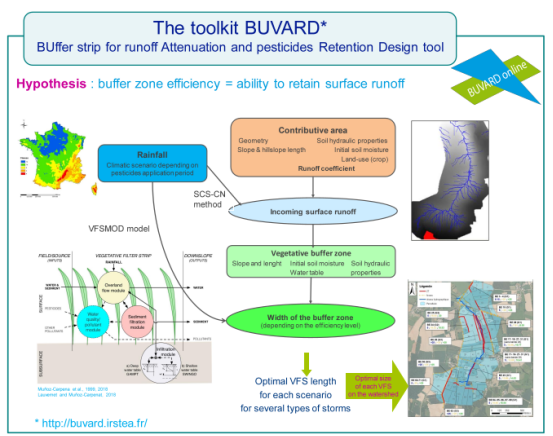

metamodeling BUVARD to bridge the gap between modeling and decision support 


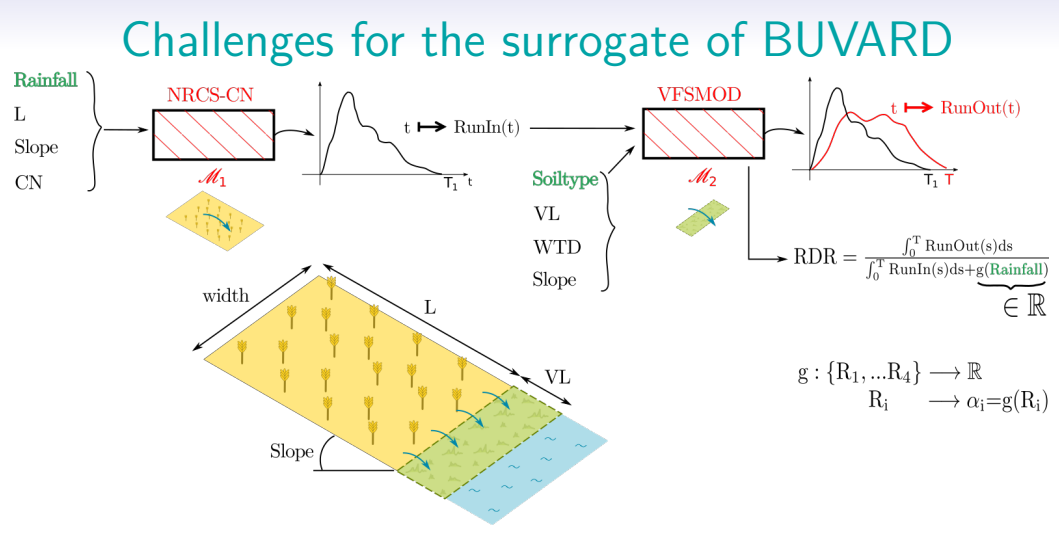

- a chain of several models

- inputs are quantitative and qualitative (categorical)

- a huge number of zero values of Runin, Runout, and then RDR

- The output variable RDR has to range between 0 and 1 


\section{Problem description}

Input

\begin{tabular}{cccc}
\hline Variable & Name & Distribution & Parameters \\
$X_{1}$ & Curve number $(\mathrm{CN})$ & Uniform & {$[63,99]$} \\
$X_{2}$ & Slope & Uniform & {$[0.1,20]$} \\
$X_{3}$ & Length & Uniform & {$[25,300]$} \\
$X_{4}$ & Rainfall type & Categorical & 4 levels with equal probability \\
\hline$X_{5}$ & Vegetative length (VL) & Uniform & {$[3,30]$} \\
$X_{6}$ & Water table depth (WTD) & Uniform & {$[50,400]$} \\
$X_{7}$ & Soil type & Categorical & 6 levels with equal probability \\
\hline
\end{tabular}

Output

- $R_{\text {in }}$ (depends on $\left.X_{1}-X_{4}\right), R_{\text {out }}$ (depends on all the inputs), Rain (depends on $X_{4}, X_{5}$ ) are recorded

- Model output: $\mathrm{RDR}=\frac{R_{\text {out }}}{R_{\text {in }}+\text { Rain }}$ which is between $[0,1]$

INRAE 


\section{Data}

2400 training data and 960 test data

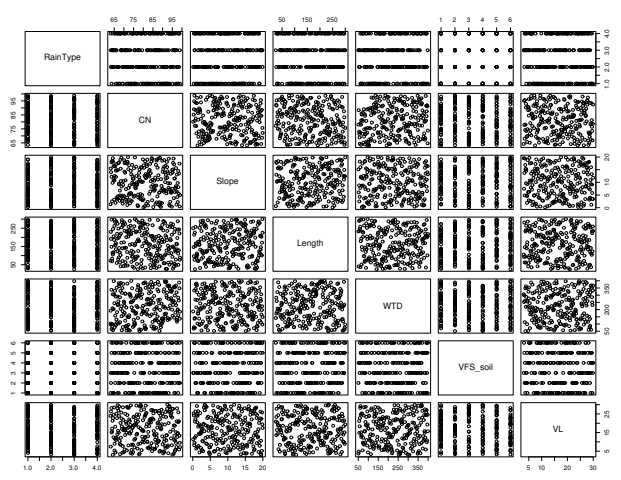

LHS Sampling approach is not too expensive, and adapted to irregular models.

Obj. = good projection properties on each axis : each 1D projection is Maximin-optimal INRAE 


\section{Metamodeling experiments}

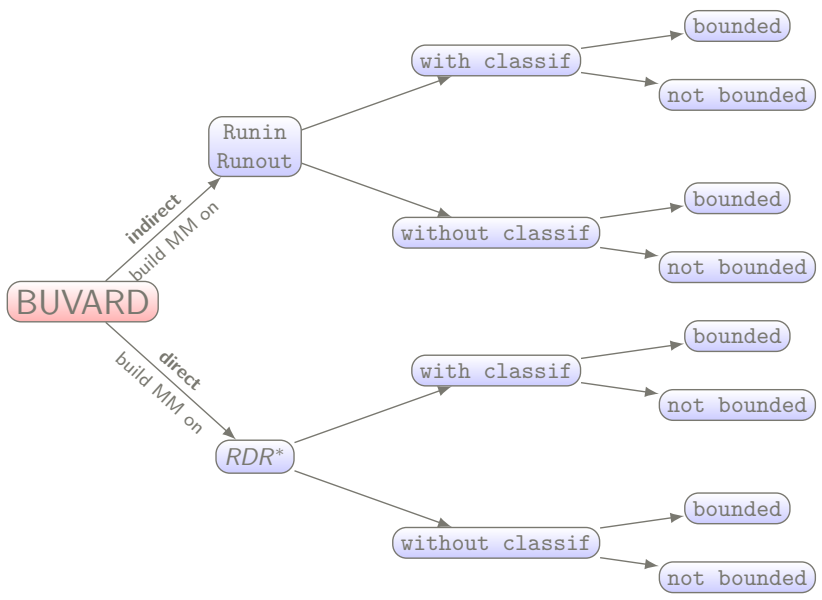

The metamodel is built using

- Gaussian Process regression / DeepGP

- Polynomial Chaos Expansion

$\rightarrow$ adapted to :

- mixed variables (quali/quanti)

- or by category

INRAE 


\section{Metamodels}

Approximation of a function $f:[0,1]^{d} \rightarrow \mathbb{R}$ from observations $\mathbf{y}=f(\mathbb{X})$ on a DoE $\mathbb{X}=\left\{\mathbf{x}^{1}, \ldots, \mathbf{x}^{n}\right\}$.

\section{Gaussian Process regression (kriging)}

- $f$ is a realization of $(Y(\mathbf{x}))_{\mathbf{x}} \sim G P(m, k(.,)$.

- Prediction : $\hat{f}(x)=\mathbb{E}(Y(\mathbf{x}) \mid Y(\mathbb{X})=\mathbf{y}\}$

- Interpolation, non parametric approach, all is in the prior.

\section{Polynomial Chaos Expansion (PCE)}

- $\hat{f}(x)=\sum_{\alpha \in \mathbb{N}^{d}} c_{\alpha} \phi_{\alpha}(\mathbf{x})$ where $\phi_{\alpha}$ are obtained by tensor product of polynomial chaos basis (Legendre, Hermite, ...).

- Estimation of $\boldsymbol{c}$ by least squares min $\|\mathbf{y}-\boldsymbol{\Psi} \boldsymbol{c}\|$, with a sparsity criterion (LASSO).

- Approximation approach. 


\section{Adaptation to categorical inputs}

Assume that the categorical variable $U$ having $K$ levels $\left\{u_{1}, \ldots, u_{K}\right\}$

Kriging : adaptation of the covariance kernel ${ }^{1}$

$$
k\left(\left(\boldsymbol{x}, u_{j}\right),\left(\boldsymbol{x}^{\prime}, u_{l}\right)\right)=k_{1}\left(\boldsymbol{x}, \boldsymbol{x}^{\prime}\right) k_{2}\left(u_{j}, u_{l}\right)
$$

$k_{2}$ is a specific covariance kernel for categorical variables, several choices are possible

Polynomial Chaos Expansion (PCE) ${ }^{2}$. The multivariate basis are given by

$$
\psi_{\boldsymbol{\alpha}}(\boldsymbol{x}, u)=\varphi_{\boldsymbol{\alpha}_{\boldsymbol{x}}}(\boldsymbol{x}) \otimes \phi_{\boldsymbol{\alpha}_{u}}(u)
$$

The estimation is done by group-LARS

$$
\hat{\boldsymbol{c}}=\arg \min _{\boldsymbol{c}}\left\|\boldsymbol{y}-\boldsymbol{\Psi}_{\boldsymbol{c}}\right\|+\nu \sum_{\mathcal{G} \in \mathcal{G}}\left\|\boldsymbol{c}_{\mathcal{G}}\right\|_{G_{\mathcal{G}}}
$$

1. See Lauvernet, C., Helbert, C. Metamodeling methods that incorporate qualitative variables for improved design of vegetative filter strips Reliability Engineering System Safety, 2020, 204, 107083

2 INRAPe Xujia Zhu,Bruno Sudret presentation, just before me! 


\section{Presence of null observations - DeepGP for non stationarity}

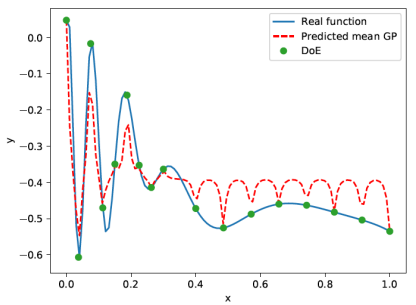

GP prediction of a non-stationary 1-D function.

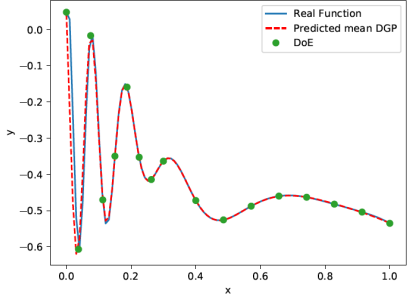

DGP prediction of a non-stationary 1-D function.

Figure: Extracted from PhD defense of Ali Hebbal 
Presence of null observations - DeepGP for non stationarity [Damianou and Lawrence, 2013]
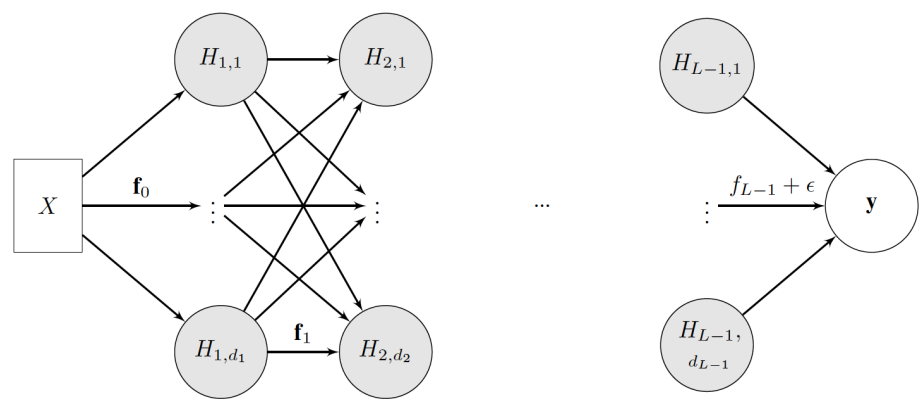

Figure: Extracted from PhD defense of Ali Hebbal 


\section{Direct $M M$ vs indirect $M M$}

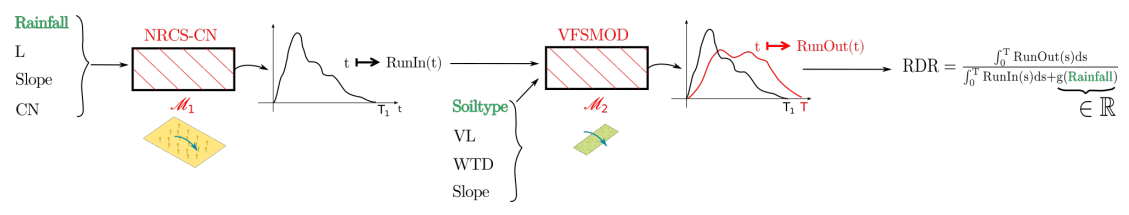




\section{Direct MM vs indirect MM}
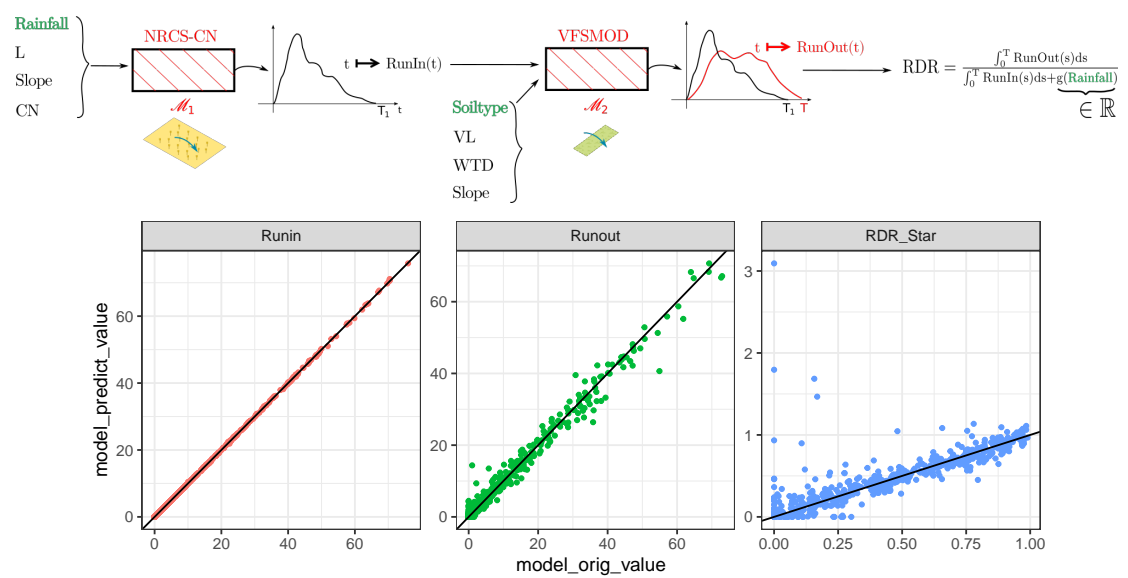

$$
\text { Kriging: } R^{2}=0.999
$$

$$
R^{2}=0.985
$$

$$
R^{2}=0.753
$$




\section{Direct $\mathrm{MM}$ vs indirect $\mathrm{MM}$}
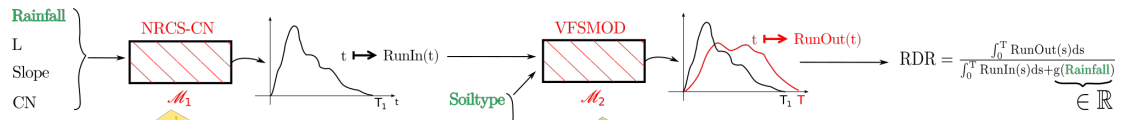

VL

WTD

Slope
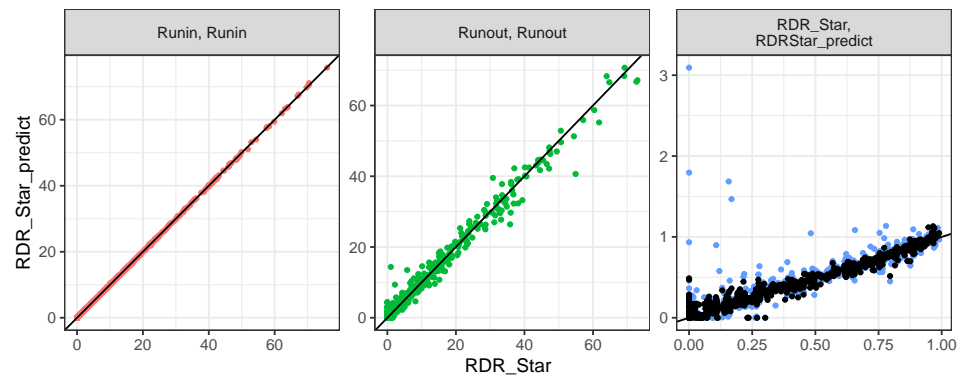

Kriging $R^{2}=0.999$

$$
R^{2}=0.985
$$$$
R^{2}=0.753 \Rightarrow 0.96
$$

$\Rightarrow$ Surrogate of the ratio is much more reliable 


\section{Direct MM vs indirect MM}
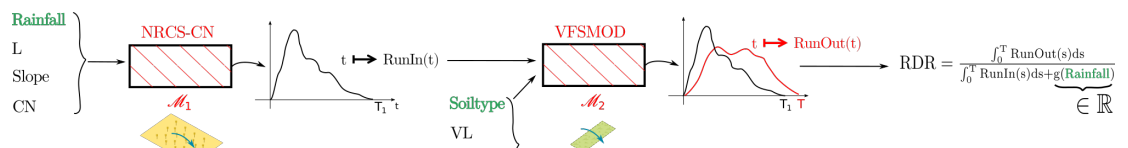

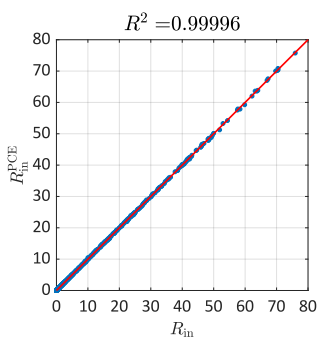

Direct PCE for Runin
WTD

Slope

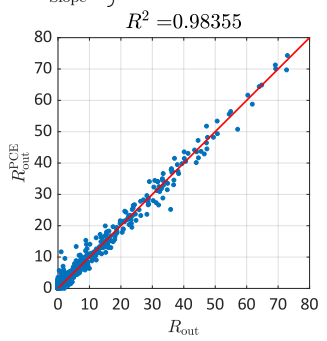

Direct PCE for Runout

$\Rightarrow$ The same for PCE ! 
Results: MM with classif / boundaries ? comparison per category

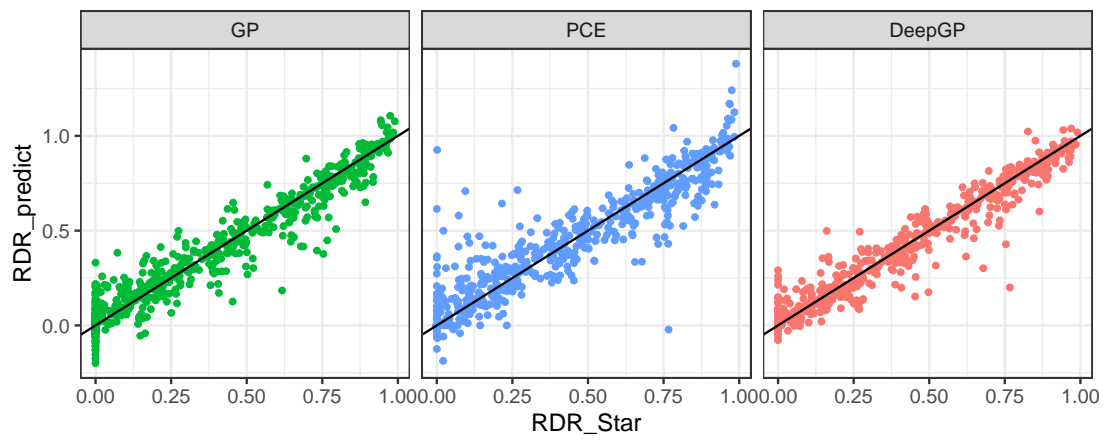

$$
R^{2}=0.951 \quad R^{2}=0.903 \quad R^{2}=0.964
$$


Results: MM with classif / boundaries ? comparison per category

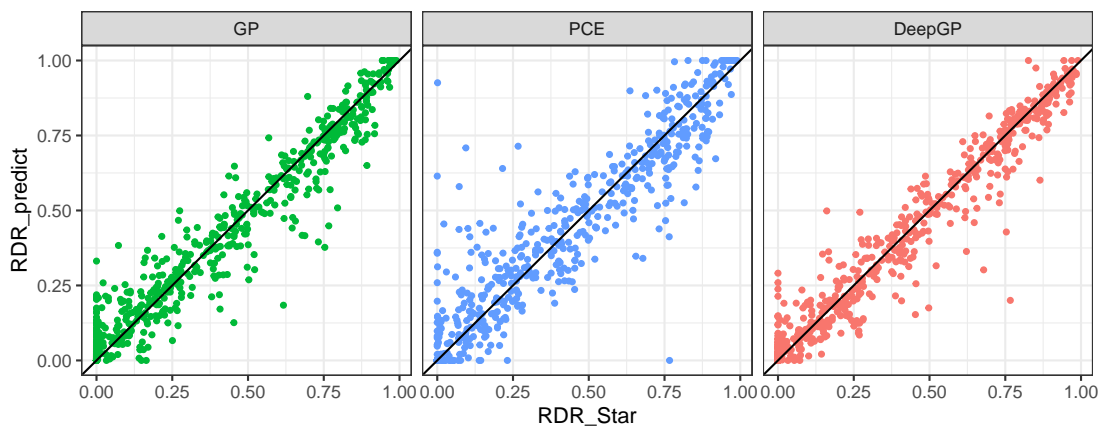

$$
\begin{aligned}
& \text { before } \quad: R^{2}=0.951 \\
& R^{2}=0.903 \\
& R^{2}=0.964 \\
& \text { bounded: } R^{2}=0.955 \\
& R^{2}=0.911 \\
& R^{2}=0.964
\end{aligned}
$$

$\Rightarrow$ DeepGP does not need any classification or boundaries 


\section{Results: mixed variables vs by category?}

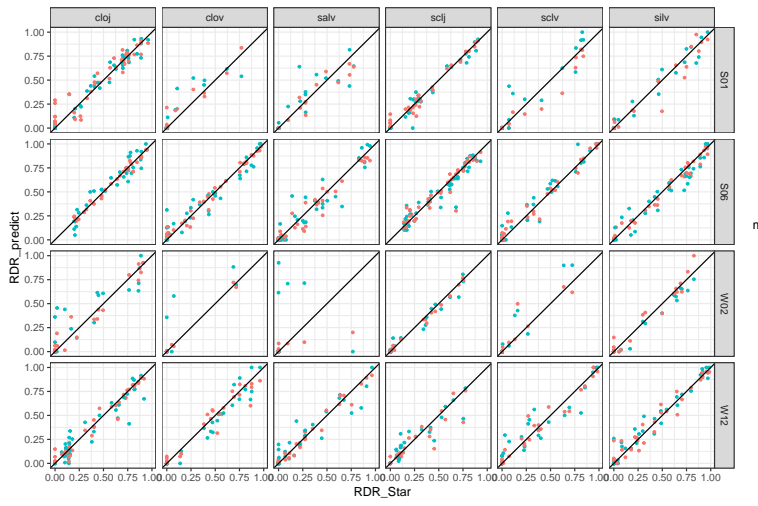

DeepGP and PCE MM by couple of category (Soil type $\times$ Rain type)

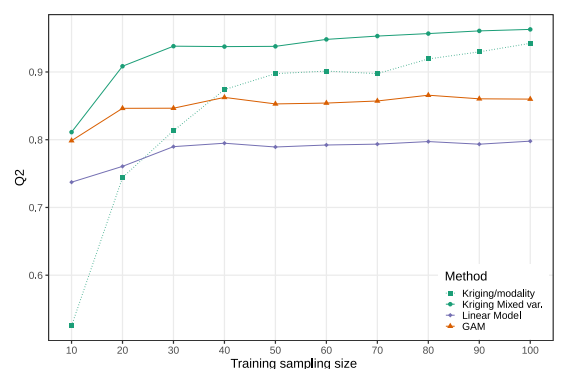

Effect of training sampling size on metamodel quality ${ }^{a}$

${ }^{a}$ extracted from Lauvernet and Helbert, 2020, RESS, 204 


\section{Results: mixed variables vs by category?}

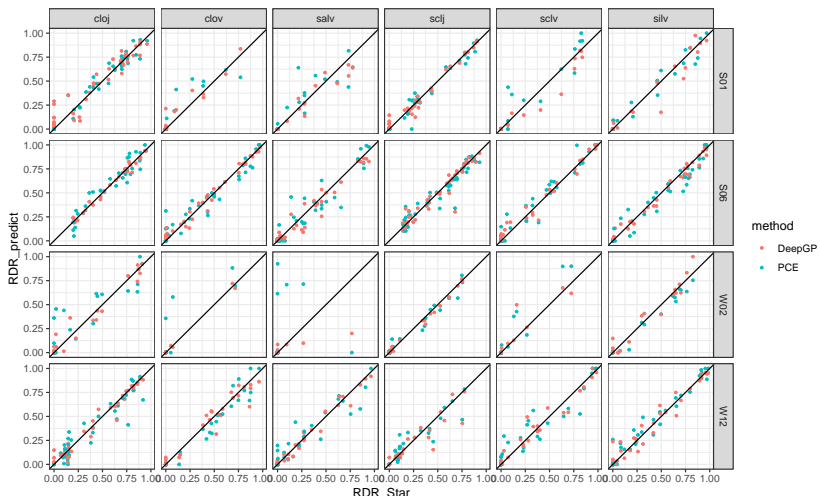

DeepGP and PCE MM by couple of category (Soil type $\times$ Rain type)

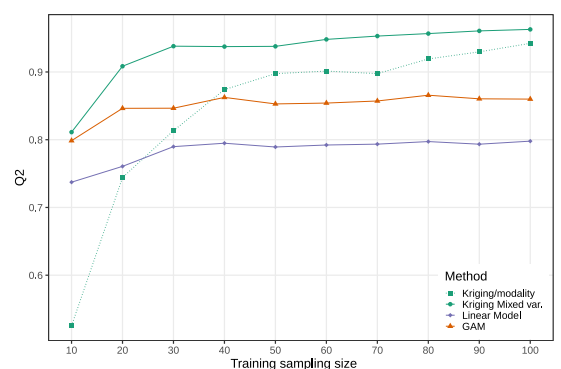

Effect of training sampling size on metamodel quality ${ }^{a}$

a extracted from Lauvernet and Helbert, 2020, RESS, 204

$\Rightarrow$ Both methods are in trouble with soils with a predominance of zeros 


\section{Results : mixed variables vs by category?}

\begin{tabular}{ccc} 
Method & $R^{2}$ per category & $R^{2}$ for mixed var. \\
\hline PCE & 0.916 & 0.966 \\
Kriging & 0.955 & 0.964 \\
DeepGP & 0.964 & - \\
\hline
\end{tabular}

$\Rightarrow$ Methods for mixed variables are more efficient and robust, and even more with smaller samplings

$\Rightarrow$ DeepGP performs well but needs repetitions for the worst soils, and is more costly numerically 


\section{Summary}

- Categorical variables were proprely taken into account by the kriging and by the PCE adaptations

- Mixed variables methods outperform the MM by category

- Classification does not improve the surrogate

- Good quality of prediction (96\% of variance is explained)

$\Rightarrow$ Next step : DeepGP for categorical variables 


\section{Thank you!}

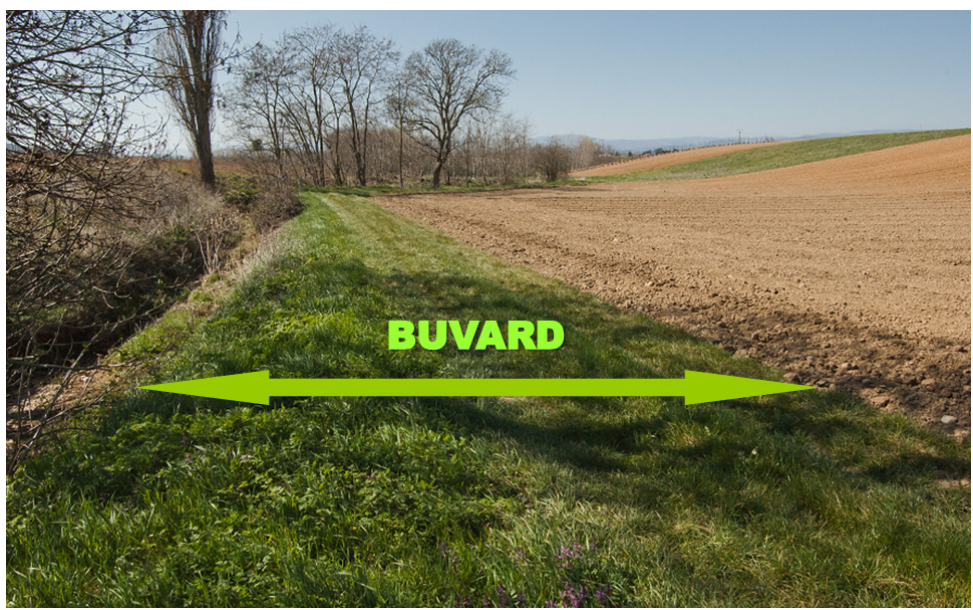

